\title{
Quantized Ultracold Neutrons in Rough Waveguides: GRANIT Experiments and Beyond
}

\author{
M. Escobar and A. E. Meyerovich \\ Department of Physics, University of Rhode Island, Kingston, RI 02881-0817, USA \\ Correspondence should be addressed to A. E. Meyerovich; alexander_meyerovich@uri.edu
}

Received 12 May 2014; Accepted 9 July 2014; Published 23 July 2014

Academic Editor: Stefan Baessler

Copyright ( 2014 M. Escobar and A. E. Meyerovich. This is an open access article distributed under the Creative Commons Attribution License, which permits unrestricted use, distribution, and reproduction in any medium, provided the original work is properly cited. The publication of this article was funded by SCOAP ${ }^{3}$.

\begin{abstract}
We apply our general theory of transport in systems with random rough boundaries to gravitationally quantized ultracold neutrons in rough waveguides as in GRANIT experiments (ILL, Grenoble). We consider waveguides with roughness in both two and one dimensions (2D and 1D). In the biased diffusion approximation the depletion times for the gravitational quantum states can be easily expressed via each other irrespective of the system parameters. The calculation of the exit neutron count reduces to evaluation of a single constant which contains a complicated integral of the correlation function of surface roughness. In the case of $1 D$ roughness (random grating) this constant is calculated analytically for common types of the correlation functions. The results obey simple scaling relations which are slightly different in $1 \mathrm{D}$ and 2D. We predict the exit neutron count for the new GRANIT cell.
\end{abstract}

\section{Introduction}

One of the most interesting recent achievements in neutron physics is a series of GRANIT [1-4] and GRANIT-inspired [5-7] experiments aimed at detection of quantization of neutron motion by the Earth gravitational field. The experiment hinges on sending a beam of ultracold neutrons between rough and flat mirrors. The rough mirror scatters away and, eventually, absorbs the neutrons in higher gravitational quantum states leaving only the particles in the lowest states which reach the neutron counter. The observation of these quantized ultralow energy levels in the peV range opens a door to using neutrons for probing weak fundamental forces. Success of these experiments stimulates similar proposals for studying atomic and antimatter beams [8-13].

Another potential application area is the use of such experiment as a test for a quantum transport theory in systems with random rough boundaries (see, for example, [14-23]; a brief review of relevant theoretical methods can be found in the beginning of [24]). The neutrons are sensitive only to geometrical and statistical properties of random surface inhomogeneities and their behavior in the rough waveguide can serve as a perfect application of our transport theory including a model-free description of the GRANIT experiments which is the main goal of this paper. Some of our earlier results for a neutron waveguide with $1 \mathrm{D}$ roughness (a mirror with random grating) can be found in $[25,26]$.

\section{General Equations}

Recently we developed a consistent perturbative approach to quantum transport along rough surfaces [24]. Within our approach, the roughness-driven transition probabilities $W_{j j^{\prime}}\left(\mathbf{q}-\mathbf{q}^{\prime}\right)$ between the states $(j, \mathbf{q}) \rightarrow\left(j^{\prime}, \mathbf{q}^{\prime}\right)$ decouple into a product of the Fourier image of the correlation function of surface roughness $\zeta\left(\mathbf{q}-\mathbf{q}^{\prime}\right)$ and the boundary values of the wave functions in the absence of roughness ( $\mathbf{q}$ is the particle momentum along the surface; $j, j^{\prime}$ are the quantum numbers describing the spatial quantization for confined motion perpendicular to the walls). In essence, the correlation function of surface roughness plays the role similar to the impurity cross-section for transport in systems with bulk impurities. 
In the case of neutron beams propagating between one rough mirror and one flat mirror, the transition probabilities have the form

$$
W_{j j^{\prime}}\left(\mathbf{q}, \mathbf{q}^{\prime}\right)=\zeta\left(\mathbf{q}-\mathbf{q}^{\prime}\right) U_{c}^{2}\left|\Psi_{j}(H)\right|^{2}\left|\Psi_{j^{\prime}}(H)\right|^{2},
$$

where $\Psi_{j}(H)$ is the value of the wave function in quantum state $j$ on the mirror in the absence of roughness and $U_{c}$ is the neutron absorption barrier for the mirror material. If the absorption barrier is very high, $U_{c} \rightarrow \infty$ (1) becomes

$$
W_{j j^{\prime}}=\frac{1}{4 m^{2}} \zeta\left(\mathbf{q}-\mathbf{q}^{\prime}\right)\left|\Psi_{j}^{\prime}(H)\right|^{2}\left|\Psi_{j^{\prime}}^{\prime}(H)\right|
$$

The wave functions $\Psi_{j}$ and the gravitational quantum states $\epsilon_{j \mathbf{q}}$ of neutrons between two horizontal mirrors are described in detail in $[25,27]$. The transition probabilities $W_{j j^{\prime}}\left(\mathbf{q}, \mathbf{q}^{\prime}\right)$ determine the collision operator in the transport equation which in this case is a set of coupled Boltzmann-like equations for the distribution functions $n_{j \mathbf{q}}$ :

$$
\begin{aligned}
\partial_{t} n_{j \mathbf{q}}=2 \pi \sum_{j^{\prime}} \int & W_{j j^{\prime}}\left(\mathbf{q}, \mathbf{q}^{\prime}\right) \\
& \times\left[n_{j^{\prime} \mathbf{q}^{\prime}}-n_{j \mathbf{q}}\right] \delta\left(\epsilon_{j \mathbf{q}}-\epsilon_{j^{\prime} \mathbf{q}^{\prime}}\right) \frac{d^{2} q^{\prime}}{(2 \pi \hbar)^{2}} .
\end{aligned}
$$

The contribution from transitions to the states within continuous spectrum above the absorption threshold $U_{c}$, for which there are no reverse processes, is negligible [25]. Integration with the energy $\delta$-function in (3) reduces these equations in the relaxation time approximation $n_{j \mathbf{q}}(\mathbf{q})=\delta\left(q-q_{j}\right) N_{j}$ to

$$
\begin{array}{r}
\partial_{t} N_{j}=\frac{m}{2 \pi} \sum_{j^{\prime}} \int d \theta\left[W_{j j^{\prime}}\left(\left|\mathbf{q}_{j}-\mathbf{q}_{j^{\prime}}\right|\right) N_{j^{\prime}}\right. \\
\left.-W_{j j^{\prime}}\left(\left|\mathbf{q}_{j}-\mathbf{q}_{j^{\prime}}\right|\right) N_{j}\right],
\end{array}
$$

where $q_{j}^{2} / 2 m=E-\epsilon_{j}$ and $\theta$ is the angle between $\mathbf{q}_{j}$ and $\mathbf{q}_{j^{\prime}}$.

We use the dimensionless variables, which are common to the field (for details see [25]). All distances are measured in units of $l_{0}$, where $l_{0}=\hbar^{2 / 3}\left(2 \mathrm{~m}^{2} \mathrm{~g}\right)^{-1 / 3} \sim 5.871 \mu \mathrm{m}$ is the size of the lowest quantum state for neutrons in the infinite gravitational trap (open geometry without an upper mirror). The dimensionless distance between the mirrors $h=H / l_{0}$ in experiment typically does not go down below 2 . The average amplitude and the correlation radius of surface roughness $\eta=\ell / l_{0}$ and $r=R / l_{0}$ are usually within the $0.1 \div 1$ range. The energies are scaled by $e_{0}=m g l_{0} \sim 0.602 \mathrm{peV} \sim$ $9.6366 \times 10^{-32} \mathrm{~J}$ which is the energy of a neutron in the lowest gravitational quantum state. The quantized levels in the gravity field $\lambda_{j}(h)=\epsilon_{j}(H) / e_{0}$ start from about 2 . In these units, the typical kinetic energy of particles in the beam $\varepsilon=E / e_{0}$ and the absorption threshold $u_{c}=U_{c} / e_{0}$ are very large, $\varepsilon, u_{c} \sim 10^{5}$, and low gravitational states $\lambda_{j} \ll \varepsilon$ are not sensitive to the behavior of the potential near the absorption threshold $u_{c}$. Velocities (momenta) along the wall $v_{j}$ in the beam direction $x$ are measured in units of $v_{0}=\sqrt{2 g l_{0}}=$ $\hbar / m l_{0} \sim 1.073 \times 10^{-2} \mathrm{~m} / \mathrm{s}, \beta_{j}=v_{j} / v_{0}=\sqrt{\varepsilon-\lambda_{j}} \equiv q_{j} l_{0}$. The characteristic times can be measured in units of $\tau_{0}$,

$$
\frac{1}{\tau_{0}}=\frac{\sqrt{2 \pi}}{4 m} \frac{\hbar}{l_{0}^{2}} \approx 1148.7 \mathrm{~s}^{-1},
$$

which provides the scale for the oscillation frequency of neutrons in the gravitational well. In the original GRANIT cell [1-3] $u_{c} / \varepsilon \sim 0.16$ and the time of flight of neutrons through the cell $t_{L}$ is $t_{L} / \tau_{0} \simeq 23$. In the new cell [28] $u_{c} \sim \varepsilon$ and $t_{L} / \tau_{0} \simeq 26$.

Diffusion of neutrons between discrete states $j$ has a strong directional bias upward, towards higher states [25]. The bias is explained by the rapid growth of the boundary values of the product of wave functions in (1) and (2) with increasing $j$ and $j^{\prime}$ (roughly, as $j^{2} j^{\prime 2}$ ). This increase in the rate of jumps $j \rightarrow j^{\prime}$ is checked by the decay of the correlation function $\zeta\left(\left|\mathbf{q}_{j}-\mathbf{q}_{j^{\prime}}\right|\right)$ at large $\left|\mathbf{q}_{j}-\mathbf{q}_{j^{\prime}}\right|$ which is determined by the value of the correlation radius $R,\left|\mathbf{q}_{j}-\mathbf{q}_{j^{\prime}}\right| \lesssim 1 / R$. In the end, the transition rates $j \rightarrow j^{\prime}$ represent, as a function of $j^{\prime}$, a relatively narrow peak around some $j_{1} \gg j$.

This strong upward bias has two consequences. First, almost all the time $\tau_{j}$ necessary for a neutron, which is initially in a low gravitational state $j$, to go up in states and disappear over the absorption barrier $u_{c}$, is spent on the first transition upwards. Further transitions to higher and higher states are going faster and faster. And second, this bias allows one to neglect the return of particles back to the lowest states. This results in decoupling of (4):

$$
\partial_{t} N_{j}=-\frac{N_{j}}{\tau_{j}}, \quad \frac{1}{\tau_{j}}=m \sum_{j^{\prime}} \int \frac{d \theta}{2 \pi} W_{j j^{\prime}}\left(\left|\mathbf{q}_{j}-\mathbf{q}_{j^{\prime}}\right|\right) .
$$

Since the high narrow peak for transitions rates $j \rightarrow j^{\prime}$ is centered around some $j_{1} \gg j$, the absorption times $\tau_{j}$ for neutrons that initially occupy low gravitational states $j$ differ from each other, according to (1), only by the values of the wave functions on the rough mirror $\Psi_{j}^{2}(H)$,

$$
\frac{1}{\tau_{j}}=\frac{b_{j}}{b_{1}} \frac{1}{\tau_{1}}, \quad b_{j}=\frac{10^{5} l_{0} \Psi_{j}^{2}(H)}{2},
$$

where $\tau_{1}$ is the depletion time for the first-the lowestgravitational state, and the coefficient $10^{5}$ is inserted purely for the computational convenience. The ratios $\tau_{j}(h) / \tau_{1}(h)$ (7) are plotted in Figure 1 for $j=2 ; 3 ; 4 ; 5$ as a function of the distance between the mirrors $h$. Note that these ratios $\tau_{j} / \tau_{1}$ in the biased diffusion approximation are the same for $1 \mathrm{D}$ and $2 \mathrm{D}$ roughness and do not depend on the roughness parameters at all. Though all the depletion times $\tau_{j}$ rapidly decrease with decreasing $h$ (see below), the ratios $\tau_{j}(h) / \tau_{1}(h)$ are increasing.

In $[29,30]$ the linewidths $1 / \tau_{j}$ were considered as independent fitting parameters. Equation (7) shows that these $\tau_{j}$ are not independent and are trivially related to each other. In the next two sections we calculate these $\tau_{j}$ and express 


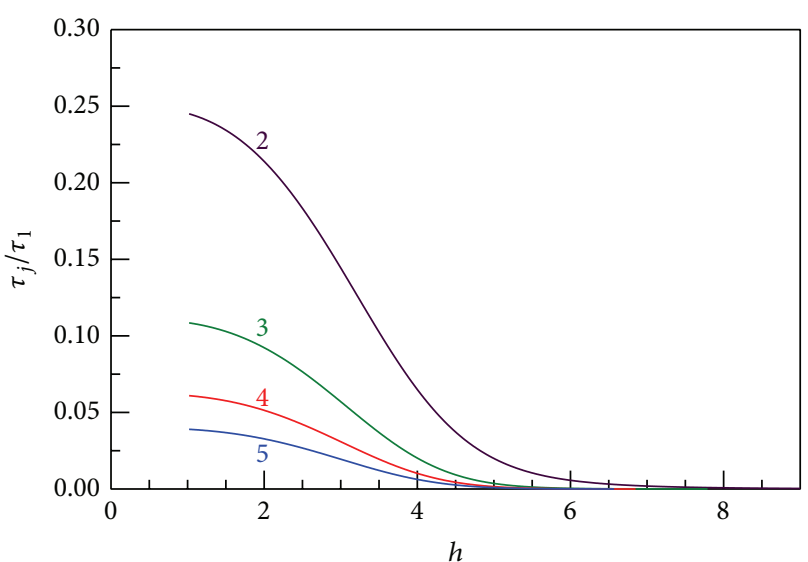

Figure 1: The ratios $\tau_{j}(h) / \tau_{1}(h)$ (7) for the lowest quantum levels $j=2 ; 3 ; 4 ; 5$ as a function of the slit width $h$. The curves are marked by the values of $j$. These functions are the same for $1 \mathrm{D}$ and $2 \mathrm{D}$ roughness and in the biased diffusion approximation do not depend on the roughness parameters.

them via the parameters of the correlation function of surface roughness.

The fact that the depletion times $\tau_{j}$ can be relatively close to each other illustrates the difficulty in observing the stepwise dependence of the exit neutron count $N_{e}$ on $h$,

$$
N_{e}=\sum N_{j}=\sum N_{j}(0) \exp \left(\frac{-L}{v_{j} \tau_{j}}\right)
$$

where $N_{j}(0)$ is the number of neutrons in state $j$ entering the waveguide of length $L$. For the lowest gravitational states the velocities $v_{j}$ are more or less the same, $v_{j} \approx v_{0} \sqrt{\varepsilon}$, and the exit neutron count is

$$
\begin{gathered}
N_{e}=\sum N_{j}(0) \exp \left(\frac{-t_{L}}{\tau_{j}}\right) \equiv \sum N_{j}(0) \exp \left(-\Phi b_{j}\right), \\
\Phi=\frac{t_{L}}{b_{1}(h) \tau_{1}(h)} .
\end{gathered}
$$

If all the states in front of the waveguide are equally populated, $N_{j}(0)=N_{0}(9)$ becomes

$$
\frac{N_{e}(h)}{N_{0}}=\sum \exp \left(-\Phi b_{j}(h)\right)
$$

and all parameters of the experimental setup collapse into the single constant $\Phi$ (or $\left.\tau_{1}\right)$. What makes $\Phi$ the most important parameter in the problem, technically more important than even $\tau_{1}$, is that it does not depend on the waveguide width $h$ while the relaxation time $\tau_{1}$ is inversely proportional to $b_{1}(h)$. Constant $\Phi$ is obviously different for waveguides with $1 \mathrm{D}$ and 2 D roughness, $\Phi_{1}$ and $\Phi_{2}$, and these two situations should be considered separately.

The value of the dimensionless constant $\Phi$ depends on the properties of the waveguide, but the moment we calculate $\Phi$ we know the dependence of the exit neutron count on the waveguide width $h$, Figure 2, irrespective of the origin of these particular values of $\Phi$.

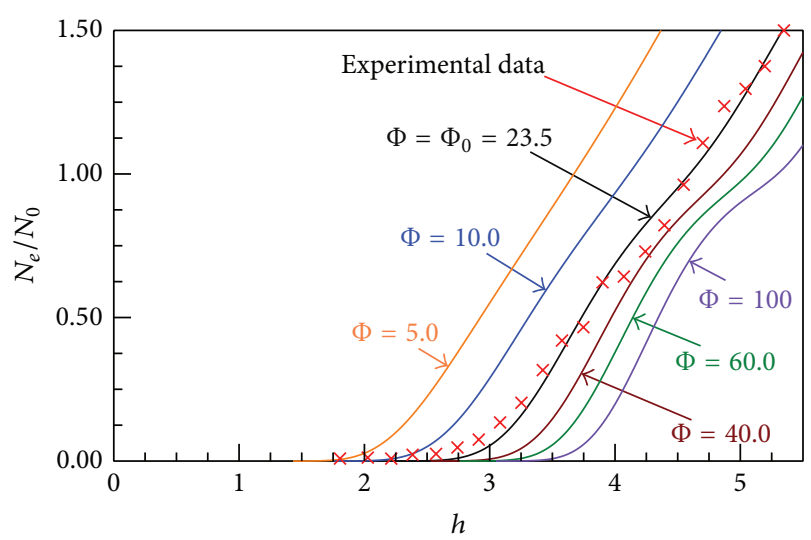

FIgURE 2: The dependence of the exit neutron count $N_{e} / N_{0}$ (11) on the waveguide width $h$ for different values of $\Phi$. The curves are marked by the values of $\Phi$. The experimental data are fitted to the curve with $\Phi=23.5$ which corresponds to $1 \mathrm{D}$ Gaussian roughness with $\eta=0.19$ and $r=1.19$.

In Figure 2 taken from [26] we plot the exit neutron count $N_{e}(h) / N_{0}$ (11) for several values of $\Phi$. The noticeable quantum steps on the curves start appearing for $\Phi>40$. These steps correspond to distinct consecutive depletions of the gravitational quantum states; at lower $\Phi$ the depletion processes overlap. The experimental data from [1-4] are fitted to the curve with $\Phi=23.5$ which assumes $1 \mathrm{D}$ Gaussian roughness with $\eta=0.19$ and $r=1.19$ (see below). The fitting parameters are the number of neutrons entering the waveguide $N_{0}$ and, to a lesser degree, the width of the waveguide which cannot be measured precisely. This is much smaller than the set of fitting parameters used in $[29,30]$. The quality of the fit is reasonably good taking into account that in earlier experiments [1-4] the absolute value of the width of the rough waveguide $h$ could not be measured with an accuracy better than $10 \%$ and that the correlation function of surface roughness and the distribution of neutrons entering the waveguide $N_{j}(0)$ have not been measured at all.

The only remaining task is to calculate $\tau_{1}$,

$$
\frac{1}{\tau_{1}}=m \sum_{j^{\prime}>1} \int \frac{d \theta}{2 \pi} W_{1 j^{\prime}}\left(\left|\mathbf{q}_{1}-\mathbf{q}_{j^{\prime}}\right|\right),
$$

and, by extension, the dimensionless parameter $\Phi(10)$.

\section{Waveguides with $1 D$ Roughness}

We start from waveguides with 1D roughness for which most of the calculations can be carried out analytically. Towards the end of the paper we will mention why $1 \mathrm{D}$ roughness is important though the existing rough mirrors exhibit $2 \mathrm{D}$ roughness [31].

It is more convenient to start not from (12) but from (6). $1 \mathrm{D}$ roughness is a random grating perpendicular to the direction of the beam and the 2D momentum both before and after scattering has only one component $p$ along 
the beam, $\mathbf{q}=(p, 0)$. The transition probabilities $W_{j j^{\prime}}$ and the distribution function have the form

$$
\begin{gathered}
W_{j j^{\prime}}\left(\mathbf{q}, \mathbf{q}^{\prime}\right)=\delta\left(q-q^{\prime}\right) W_{j j^{\prime}}\left(p, p^{\prime}\right), \\
n_{j}(\mathbf{q})=\frac{(2 \pi)^{2}}{L_{p} L_{q}} \delta(q) \delta\left(p-p_{j}\right) N_{j},
\end{gathered}
$$

where $N_{j}$ is the number of particles in state $j$ per unit length of the beam and $p_{j}=\sqrt{E-\epsilon_{j}}$, and $E$ is the overall energy of the neutrons. Then (6) reduce to

$$
\partial_{t} N_{j}=-\frac{N_{j}}{\tau_{j}}, \quad \frac{1}{\tau_{j}}=\sum_{j^{\prime}>j} \frac{W_{j j^{\prime}}\left(p_{j}-p_{j^{\prime}}\right)}{v_{j^{\prime}}} .
$$

In our dimensionless variables the scattering probabilities (1) and (13) in (15) obtain the form

$$
\begin{gathered}
\frac{1}{\tau_{j}}=\frac{1}{\tau_{0}} \sum_{j^{\prime}>j} \frac{w_{j j^{\prime}}\left(p_{j}-p_{j^{\prime}}\right)}{v_{j^{\prime}}}, \\
w_{j j^{\prime}}=\frac{4 \times 10^{-10} u_{c}^{2} \eta^{2} r \psi_{1}\left(y_{j j^{\prime}}\right) b_{j} b_{j^{\prime}}}{\beta_{j}}, \\
y_{j j^{\prime}}=r\left(\beta_{j}-\beta_{j^{\prime}}\right),
\end{gathered}
$$

where $\psi_{1}$ stands for the dimensionless part of the Fourier image of $1 D$ correlation function of surface roughness:

$$
\zeta_{1}\left(p_{j}-p_{j^{\prime}}\right)=\sqrt{2 \pi} l_{0}^{3} \eta^{2} r \psi_{1}\left(y_{j j^{\prime}}\right)
$$

the lower index 1 indicates that this equation describes the case of $1 \mathrm{D}$ roughness.

Since the transition rate $j \rightarrow j^{\prime}$ rapidly increases with increasing $j^{\prime}$, only the terms with large $j^{\prime}$ make a noticeable contribution to the sum (16) which can be replaced by the integration:

$$
\begin{aligned}
\frac{\tau_{0}}{\tau_{1}}=2 \times 10^{-5} u_{c}^{2}\left(\frac{\eta^{2}}{r^{2}}\right) b_{1}(h) F_{1}(r, h), \\
F_{1}(r, h)=2 \times 10^{-5} r^{3} \varepsilon^{-1 / 2} \sum b_{j} \psi_{1}\left(y_{1 j}\right) \\
=\frac{2 r^{3} \varepsilon}{\pi u_{c}} \int_{0}^{1} d z \cdot z^{2} \psi_{1}(y), \\
y=r \varepsilon^{1 / 2}\left(1-\sqrt{1-z^{2}}\right) .
\end{aligned}
$$

In these notations our main parameter $\Phi_{1}$ obtains a very simple form:

$$
\begin{gathered}
\Phi_{1}=A_{1} \eta^{2} r \int_{0}^{1} d z \cdot z^{2} \psi_{1}(y), \\
A_{1}=\frac{4 \times 10^{-5}}{\pi} \frac{t_{L}}{\tau_{0}} \frac{\varepsilon}{u_{c}}
\end{gathered}
$$

and the problem reduces to the integrations (21) and (23) of the correlation functions of various functional forms.

If, as it is often assumed, the correlation function is Gaussian, $\zeta_{1}(x / r)=l_{0}^{2} \eta^{2} \exp \left(-x^{2} / 2 r^{2}\right)$, its Fourier image is also Gaussian, $\psi_{1}(y)=\exp \left(-y^{2} / 2\right)$ and

$$
\frac{\Phi_{1}}{A_{1} \eta^{2}}=\frac{1}{3 \sqrt{r}}\left(\frac{8}{\varepsilon}\right)^{3 / 4} \Gamma\left(\frac{7}{4}\right) .
$$

This equation has been used in [26] when fitting the experimental data from [1-4] (see Figure 2). The only information we have about the mirror roughness in those earlier experiments is that the average lateral size of inhomogeneities is of the order 1.19 and the average amplitude is approximately 0.119 . Assuming that these numbers give the values of $r$ and $\eta$ and that we are dealing with the 1D Gaussian roughness, (25) yields $\Phi_{1} \sim 23.5$. This value is used in the fit in Figure 1. Since these assumptions are somewhat arbitrary, the quality of the fit is actually much better than one might expect with all the uncertainties.

The Fourier image of a power law correlation function,

$$
\zeta_{1}\left(\frac{x}{r}\right)=l_{0}^{2} \eta^{2} \frac{2 \mu}{\left(1+x^{2} / r^{2}\right)^{1+\mu}},
$$

behaves like an exponential function:

$$
\begin{gathered}
\psi_{1}(y)=\frac{y^{\mu} K_{\mu}(y)}{2^{\mu-1} \Gamma(\mu)}, \\
\frac{\Phi_{1}}{A_{1} \eta^{2}}=\sqrt{\frac{\pi}{r}} \varepsilon^{-3 / 4} 2^{1-\mu} \mu .
\end{gathered}
$$

In the opposite case, when the power spectrum of roughness $\psi_{1}(y)$ is given by a power law function,

$$
\psi_{1}(y)=\frac{1}{\left(1+y^{2}\right)^{1+\lambda}}
$$

the correlation function itself behaves exponentially:

$$
\zeta_{1}\left(\frac{x}{r}\right)=\frac{l_{0}^{2} \eta^{2}(x / r)^{\lambda} K_{\lambda}(x / r)}{2^{\lambda} \Gamma(1+\lambda)}
$$

and the constant $\Phi_{1}$ has the form

$$
\frac{\Phi_{1}}{A_{1} \eta^{2}}=\frac{1}{3 \sqrt{r}}\left(\frac{4}{\varepsilon}\right)^{3 / 4} \frac{\Gamma(\lambda+1 / 4)}{\Gamma(\lambda+1)} .
$$

The purely exponential correlation functions in configuration or momentum spaces emerge from (30) or (27) when $\lambda=1 / 2$ or $\mu=1 / 2$ :

$$
\exp (-s)=\sqrt{\frac{2 s}{\pi}} K_{1 / 2}(s) .
$$

The parameter $\Phi_{1}$ and the relaxation times $\tau_{j}$ exhibit a universal dependence on the amplitude $\eta$ and the correlation 


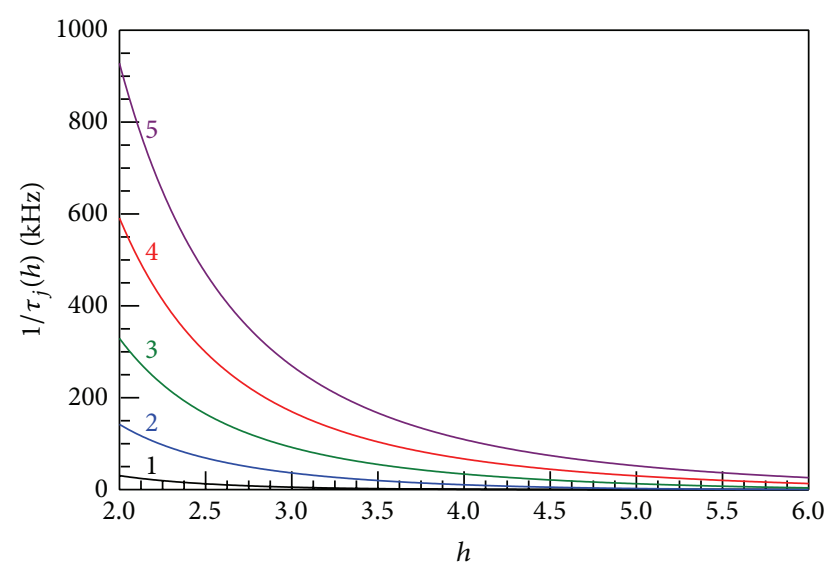

Figure 3: The inverse depletion times $1 / \tau_{j}$ for the lowest gravitational states $j=1 ; 2 ; 3 ; 4 ; 5$ for a waveguide with $1 \mathrm{D}$ exponential roughness with $\eta=r=1$. The curves are marked by the values of $j$. The vertical scale is in $\mathrm{kHz}$. Equation (33) extends the results to other values of $\eta$ and $r$.

radius $r$ of the surface roughness and on the overall kinetic energy of particles in the beam $\varepsilon$ :

$$
\Phi_{1}, \frac{1}{\tau_{j}} \propto \frac{\eta^{2}}{r^{1 / 2} \varepsilon^{3 / 4}} .
$$

This scaling is precise only at $u_{c} \rightarrow \infty$. For finite $u_{c}$ the powers of $r$ and $\varepsilon$ are slightly different (e.g., at $u_{c} \approx 10^{5}$ the scaling for the exponential correlator is $r^{-0.493}$ ).

These equations allow one to find the depletion times $\tau_{j}$ for each gravitational state, (7) and (10). Figure 3 presents inverse depletion times $1 / \tau_{j}(h)$ in $\mathrm{kHz}$ for $j=1 ; 2 ; 3 ; 4 ; 5$ in a waveguide with exponential roughness $\eta=r=1$, (31) and (32). The values of the neutron velocity and time of flight entering $A_{1}$ (24) correspond to the original GRANIT cell [1$4]: t_{L} / \tau_{0} \approx 23, u_{c} / \varepsilon \approx 0.16$. The scaling (33) allows one to get $1 / \tau_{j}$ for other values of $\eta, r$. The depletion becomes faster or slower depending on the value of $\eta^{-2} r^{1 / 2}$.

The depletion times $\tau_{j}$ determine both the disappearance of neutrons and broadening of the gravitational energy states $\epsilon_{j}+i \hbar / \tau_{j}(h)$ with decreasing width of the waveguide $h$. The broadening of the state $j$ increases dramatically and rapidly reaches the separation between the states after the width $h$ becomes smaller than some critical value $h_{j}$. Below $h_{j}$ the gravitational state $\epsilon_{j}$ looses its discrete quantum nature; this happens simultaneously with depletion of the neutron population of this state.

\section{Waveguides with 2D Roughness}

In contrast to systems with $1 \mathrm{D}$ roughness, most of the calculations in $2 \mathrm{D}$ cases can be done only numerically. We start from (12) which in our notations acquires the form similar to (20):

$$
\frac{\tau_{0}}{\tau_{1}}=2 \times 10^{-5} u_{c}^{2}\left(\frac{\eta^{2}}{r^{2}}\right) b_{1} F_{2}(r, h),
$$

where

$$
\begin{gathered}
F_{2}(r, h)=10^{-5} \sqrt{\frac{2}{\pi}} r^{4} \sum b_{j} \psi_{2}^{(0)}\left(Q_{1}, Q_{j}\right), \\
Q_{j}=q_{j} r, \quad q_{j}=\sqrt{\varepsilon-\lambda_{j}} .
\end{gathered}
$$

After replacing the summation by the integration, we get equations similar to (23) and (24):

$$
\begin{gathered}
\Phi_{2}=A_{2} \eta^{2} r^{2} \int_{0}^{1} d z \cdot z^{2} \psi_{2}^{(0)}\left(y_{1}, \tilde{y}\right), \\
y_{1}=r \varepsilon^{1 / 2}, \quad \tilde{y}=r \varepsilon^{1 / 2} \sqrt{1-z^{2}} \\
A_{2}=10^{-5}\left(\frac{2}{\pi}\right)^{3 / 2} \frac{t_{L}}{\tau_{0}} u_{c} \varepsilon^{3 / 2} .
\end{gathered}
$$

The calculation of the zeroth angular harmonic of the $2 \mathrm{D}$ correlation function in momentum space $\psi_{2}^{(0)}$ can be done analytically [32] for the Gaussian

$$
\psi_{2}^{(0)}\left(Q, Q^{\prime}\right)=4 \pi e^{-Q Q^{\prime}} I_{0}\left(Q Q^{\prime}\right) e^{-\left(Q-Q^{\prime}\right)^{2} / 2}
$$

and exponential

$$
\begin{aligned}
\psi_{2}^{(0)}\left(Q, Q^{\prime}\right) & =\frac{8 E(\Omega)}{\left[1+\left(Q-Q^{\prime}\right)^{2}\right] \sqrt{1+\left(Q+Q^{\prime}\right)^{2}}}, \\
\Omega & =2 \sqrt{\frac{Q Q^{\prime}}{\left[1+\left(Q+Q^{\prime}\right)^{2}\right]}}
\end{aligned}
$$

correlation functions. Even in these cases all further calculations should be done numerically.

In contrast to the earlier experiments, the roughness correlation function for the ongoing GRANIT experiments in a new cell has been measured [31]. This correlation function is very close to the isotropic $2 \mathrm{D}$ exponential function with the correlation radius $r=0.65$ and amplitude $\eta=1.02$ while the time of flight $t_{L} / \tau_{0} \simeq 26$. This roughness yields $\Phi_{2}=5.22 \times 10^{3}$. The numerical examples below assume this setup. Our results for other correlation functions can be found in [31] in which we discuss the identification of the correlation function for the new rough mirror.

As in the case of $1 \mathrm{D}$ roughness, both $\Phi$ and $1 / \tau_{j}$ remain proportional to $\eta^{2}$. This scaling is obvious. The dependence on the correlation radius is more elusive and we cannot get an analytical expression similar to (33). The reason is the presence of $r \varepsilon^{1 / 2}$ in the argument of $\psi_{2}^{(0)}(40)$ in the integrand in (36). Our numerical data show that the scaling for $r>0.3$ remains similar to (33):

$$
\Phi, \frac{1}{\tau_{j}} \propto \eta^{2} r^{\gamma} \varepsilon^{\delta}
$$

At $u_{c} \rightarrow \infty$ the index, $\gamma=-1 / 2$, the same as in the case of $1 \mathrm{D}$ roughness. The deviation from $\gamma=-1 / 2$ at finite $u_{c}$ is more pronounced than in $1 \mathrm{D}$ case: at $u_{c}=10^{5}$, as in experiment, 


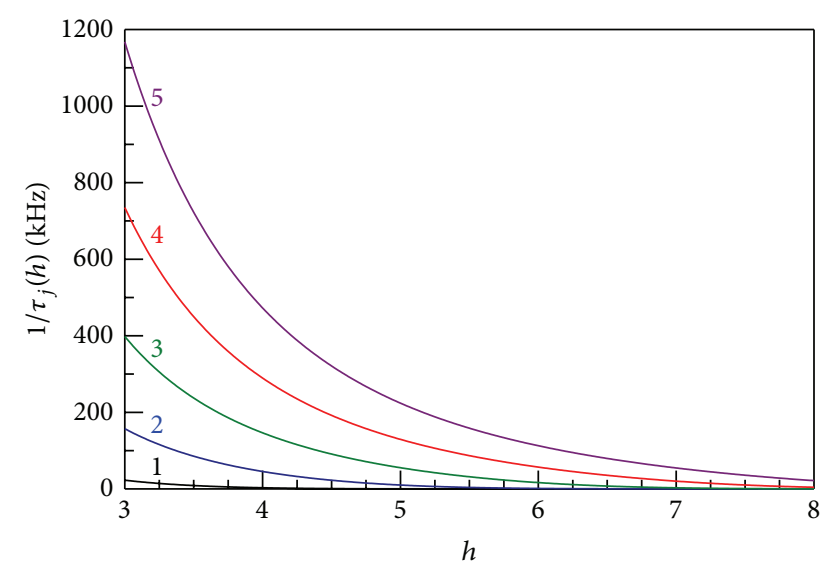

FIgURE 4: The same as Figure 3 for $2 \mathrm{D}$ exponential roughness in the new cell.

the index $\gamma$ becomes -0.465 instead of -0.493 as in $1 D$. The scaling index for energy in the $2 \mathrm{D}$ case is obviously different from (33) because of different dependencies on velocities in the integrand. Instead of $\delta=-3 / 4$ as in (33), we find $\delta \simeq$ -1.165 though, in contrast to $\gamma$, the quality of the fit is not very good.

Figure 4 presents inverse depletion times $1 / \tau_{j}(h)$ in $\mathrm{kHz}$ for $j=1 ; 2 ; 3 ; 4 ; 5$ in a waveguide with $2 \mathrm{D}$ exponential roughness $\eta=r=1,(34)$, (36), and (40). The values of the neutron velocity and time of flight entering $A_{2}$ (38) correspond to the new GRANIT cell [1-4]: $t_{L} / \tau_{0} \approx 26, u_{c} / \varepsilon \sim$ 1 . The scaling $\eta^{2} r^{\mu}(41)$ allows one to get $1 / \tau_{j}$ for other values of $\eta, r$.

The critical values of $h_{j}$, below which the depletion and the broadening of the gravitational state $\epsilon_{j}(h)$ explode, are slightly larger than those for the 1D roughness. This is explained by the role of the sideway scattering which is absent in the $1 \mathrm{D}$ case. Otherwise, Figures 3 and 4 are similar.

Our prediction for the neutron count $N_{e}(h) / N_{0}$ for the new GRANIT cell with the 2D exponential roughness with $\eta=1.03$ and $r=0.65\left(\Phi_{2}=5.22 \times 10^{3}\right)$ is given in Figure 5 . The curve exhibits much more pronounced quantum steps than those in Figure 1. It is worth mentioning that the neutron count $N_{e}$ is much less sensitive to the value of $\Phi$ when $\Phi$ is in $(2 \div 8) \times 10^{3}$ range than when it is in the range $5 \div 40$ as in Figure 1 .

The presence of well-developed steps on the curve, which correspond to consecutive depletion of the gravitational quantum states, is explained mostly by a relatively large amplitude of roughness $\eta$, several times bigger than for the older cell. This large value of the amplitude of roughness $\eta$ presents challenges for both theory and experiment and can potentially degrade the accuracy of information extracted from the experimental data. On a theoretical side, the main assumption of the theory, $\eta \ll r, h$, is violated. For experiment, the large amplitude of roughness means that it becomes virtually impossible to accurately measure the distance between the mirrors $h$. It also means that the energy levels become broad and not very well defined even if one disregards the interlevel transitions. The most likely

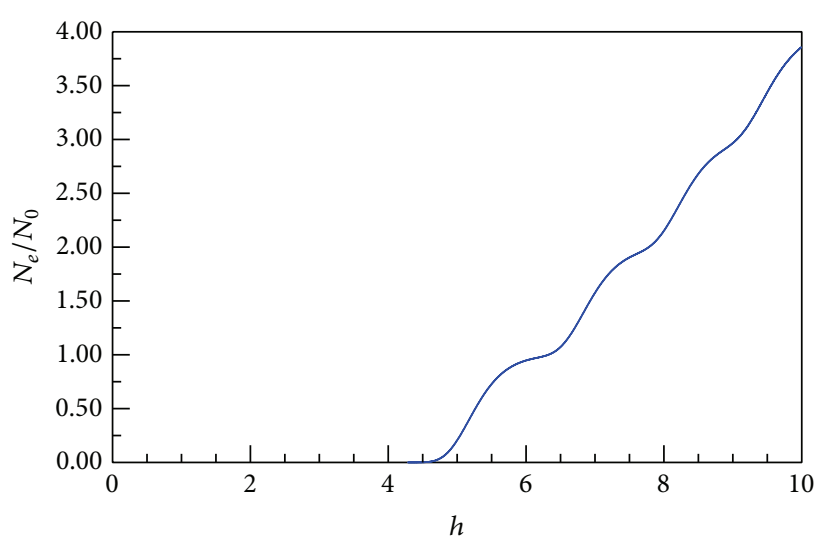

FIGURE 5: The prediction for the neutron count for the new waveguide with $2 \mathrm{D}$ exponential roughness with parameters $r=0.65$, $\eta=1.02$.

consequence of all these factors would be some smearing of the steps in comparison to those in Figure 5.

\section{Conclusions}

In summary, we developed a quantitative theory of propagation of ultracold neutrons through a rough waveguide. The immediate applications are the ongoing GRANIT experiments at ILL (Grenoble) aimed at analysis of quantization of neutrons by the gravity field. There are also other experimental groups exploring similar setups. If successful, these experiments will produce neutrons in well defined ultralow energy states in the peV range which can be used for precise measurements of fundamental forces.

We analyzed waveguides with $1 \mathrm{D}$ and $2 \mathrm{D}$ roughness. The ratios of the depletion times (line broadenings) in the biased diffusion approximation were the universal functions of the waveguide width and did not depend on the waveguide parameters. All relevant waveguide and roughness parameters collapsed into a single constant (essentially, a linewidth of the lowest quantum state), which was responsible for the exit neutron count. This constant strongly depended on the functional form of the roughness correlation function. We calculated this constant for various waveguides. In waveguides with $1 \mathrm{D}$ roughness the calculations could be carried out analytically for the most common types of the correlation functions; the $2 \mathrm{D}$ calculations were mostly numerical.

Our results were in good agreement with earlier experimental data despite the lack of experimental information about many important parameters. The predicted neutron count for the new experimental setup, for which the roughness profile was accurately measured, exhibited well-developed quantum step corresponding to consecutive depletion of the lower and lower gravitational states. Large amplitude of roughness in this setup could degrade the usability of the results. One of the possible ways to circumvent these difficulties and produce a much more controllable environment would be the use of a radically new design for a rough mirror which we called an Ising mirror [31, 33]. 


\section{Conflict of Interests}

The authors declare that there is no conflict of interests regarding the publication of this paper.

\section{Acknowledgment}

The results of this paper were reported at GRANIT-2014 Workshop (Les Houches, France, March 2-7, 2014). One of the authors (A. E. Meyerovich) is grateful to the organizers and other members of GRANIT collaboration for support and hospitality during the workshop.

\section{References}

[1] V. V. Nesvizhevsky, H. G. Börner, A. K. Petukhov et al., "Quantum states of neutrons in the Earth's gravitational field," Nature, vol. 415, no. 6869, pp. 297-299, 2002.

[2] V. V. Nesvizhevsky, A. K. Petoukhov, H. G. Börner et al., "Measurement of quantum states of neutrons in the Earth's gravitational field," Physical Review D, vol. 67, Article ID 102002, 2003.

[3] V. V. Nesvizhevsky, A. K. Petukhov, H. G. Börner et al., "Study of the neutron quantum states in the gravity field," European Physical Journal C, vol. 40, no. 4, pp. 479-491, 2005.

[4] V. V. Nesvizhevsky, "Near-surface quantum states of neutrons in the gravitational and centrifugal potentials," Physics-Uspekhi, vol. 53, no. 7, pp. 645-675, 2010.

[5] T. Jenke, P. Geltenbort, H. Lemmel, and H. Abele, "Realization of a gravity-resonance-spectroscopy technique," Nature Physics, vol. 7, no. 6, pp. 468-472, 2011.

[6] L. A. Chizhova, S. Rotter, T. Jenke et al., "Vectorial velocity filter for ultracold neutrons based on a surface-disordered mirror system," Physical Review E, vol. 89, Article ID 032907, 2014.

[7] G. Ichikawa, S. Komamiya, Y. Kamiya et al., "Observation of the spatial distribution of gravitationally bound quantum states of ultracold neutrons and its derivation using the Wigner function," Physical Review Letters, vol. 112, Article ID 071101, 2014.

[8] A. Y. Voronin, P. Froelich, and V. V. Nesvizhevsky, "Gravitational quantum states of antihydrogen," Physical Review A, vol. 83, Article ID 032903, 2011.

[9] A. Y. Voronin, V. V. Nesvizhevsky, and S. Reynaud, "Whispering-gallery states of antihydrogen near a curved surface," Physical Review A, vol. 85, Article ID 014902, 2012.

[10] G. Dufour, P. Debu, A. Lambrecht, V. V. Nesvizhevsky, S. Reynaud, and A. Y. Voronin, "Shaping the distribution of vertical velocities of antihydrogen in GBAR," The European Physical Journal C, vol. 74, Article ID 2731, 2014.

[11] A. Y. Voronin et al., Advances in High Energy Physics, 2014, In press.

[12] G. Chardin, P. Grandemange, D. Lunney et al., "Proposal to measure the gravitational behaviour of antihydrogen at rest," Tech. Rep. CERN-SPSC-2011-029. SPSC-P-342, CERN, Geneve, Switzerland, 2011.

[13] P. Crivelli et al., "Can we observe the gravitational quantum states of positronium?," Advances in High Ednergy Physics. In press.

[14] F. G. Bass and I. M. Fuks, Wave Scattering from Statistically Rough Surfaces, Pergamon Press, 1979.
[15] J. A. DeSanto and D. S. Brown, Analytical Techniques for Multiple Scattering from Rough Surfaces (Progress in Optics, v. 23 ), E. Wolf, Amsterdam, The Netherlands, 1986.

[16] J. A. Ogilvy, Theory of Wave Scattering from Random Surfaces, Adam Hilger, Bristol, UK, 1991.

[17] A. G. Voronovich, Wave Scattering at Rough Surfaces, Springer, Berlin, Germany, 1994.

[18] G. Fishman and D. Calecki, "Surface-induced resistivity of ultrathin metallic films: a limit law," Physical Review Letters, vol. 62, no. 11, pp. 1302-1305, 1989.

[19] G. Fishman and D. Calecki, "Influence of surface roughness on the conductivity of metallic and semiconducting quasi-twodimensional structures," Physical Review B, vol. 43, no. 11, p. 11581, 1991

[20] Z. Tesanovic, M. V. Jaric, and S. Maekawa, "Quantum transport and surface scattering," Physical Review Letters, vol. 57, p. 2760, 1986.

[21] N. Trivedi and N. W. Ashcroft, "Quantum size effects in transport properties of metallic films," Physical Review B, vol. 38, no. 17, pp. 12298-12309, 1988.

[22] A. E. Meyerovich and S. Stepaniants, "Transport phenomena at rough boundaries," Physical Review Letters, vol. 73, no. 2, pp. 316-319, 1994.

[23] A. E. Meyerovich and S. Stepaniants, "Transport in channels and films with rough surfaces," Physical Review B, vol. 51, no. 23, pp. 17116-17130, 1995.

[24] A. E. Meyerovich and A. Stepaniants, "Quantized systems with randomly corrugated walls and interfaces," Physical Review B, vol. 60, no. 12, pp. 9129-9144, 1999.

[25] R. Adhikari, Y. Cheng, A. E. Meyerovich, and V. V. Nesvizhevsky, "Quantum size effect and biased diffusion of gravitationally bound neutrons in a rough waveguide," Physical Review A, vol. 75, no. 6, Article ID 063613, 2007.

[26] M. Escobar and A. E. Meyerovich, "Beams of gravitationally bound ultracold neutrons in rough waveguides," Physical Review A, vol. 83, Article ID 033618, p. 033618, 2011.

[27] A. E. Meyerovich and V. V. Nesvizhevsky, "Gravitational quantum states of neutrons in a rough waveguide," Physical Review A, vol. 73, Article ID 063616, 2006.

[28] V. V. Nesvizhevsky, "privatecommunication".

[29] A. Y. Voronin, H. Abele, S. Baeßler et al., "Quantum motion of a neutron in a waveguide in the gravitational field," Physical Review D, vol. 73, no. 4, Article ID 044029, 2006.

[30] S. Baessler, M. Beau, M. Kreuz et al., "The GRANIT spectrometer," Comptes Rendus Physique, vol. 12, pp. 707-728, 2011.

[31] M. Escobar, F. Lamy, A. E. Meyerovich, and V. V. Nesvizhevsky, Advances in High Energy Physics, 2014, In press.

[32] A. E. Meyerovich and I. V. Ponomarev, "Surface roughness and size effects in quantized films," Physical Review B, vol. 65, Article ID 155413, 2002.

[33] M. Escobar and A. E. Meyerovich, Journal of Experimental and Theoretical Physics. In press. 

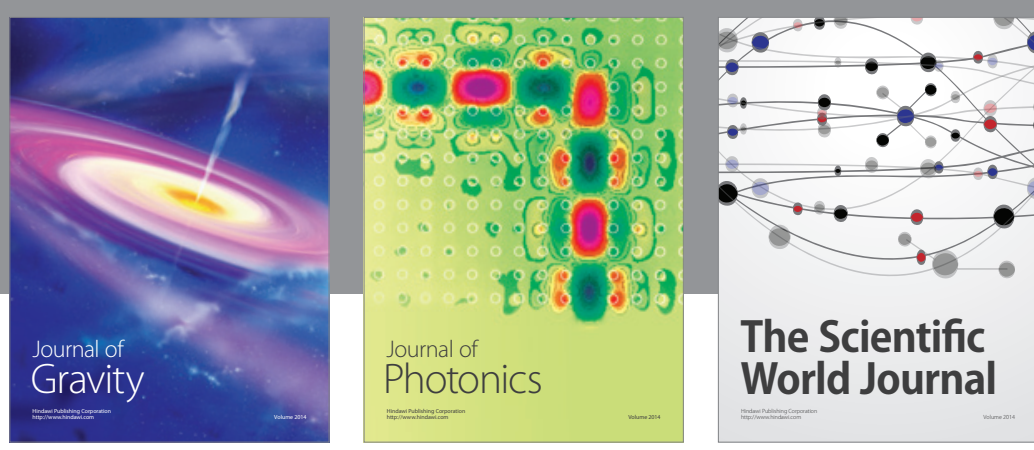

The Scientific World Journal
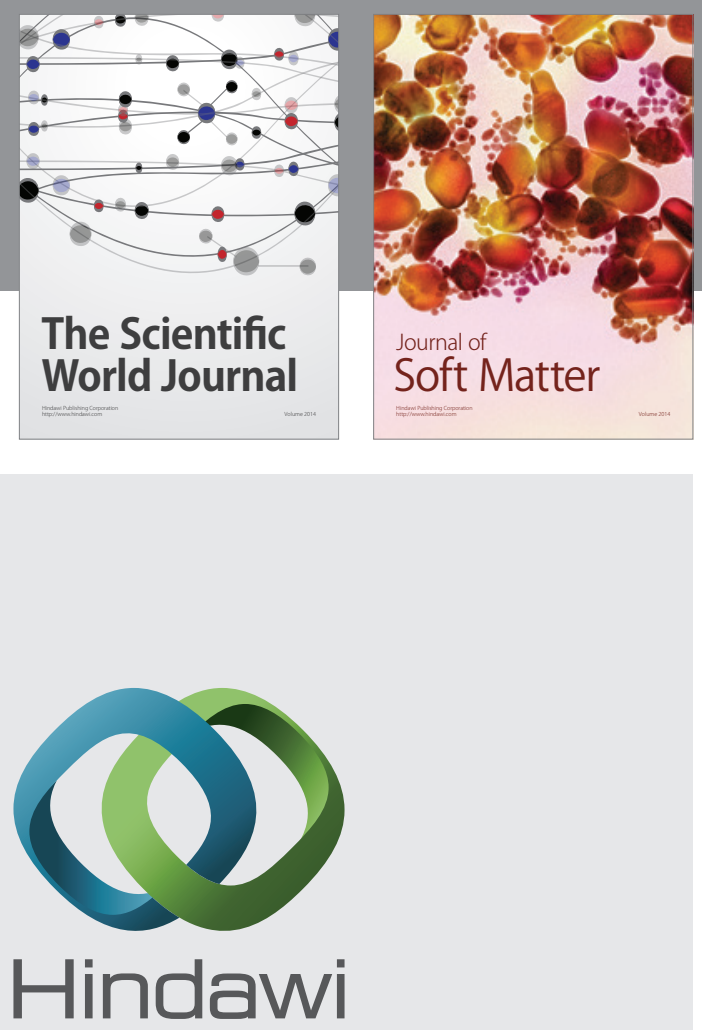

Submit your manuscripts at

http://www.hindawi.com

nternational Journal of

Statistical Mechanics
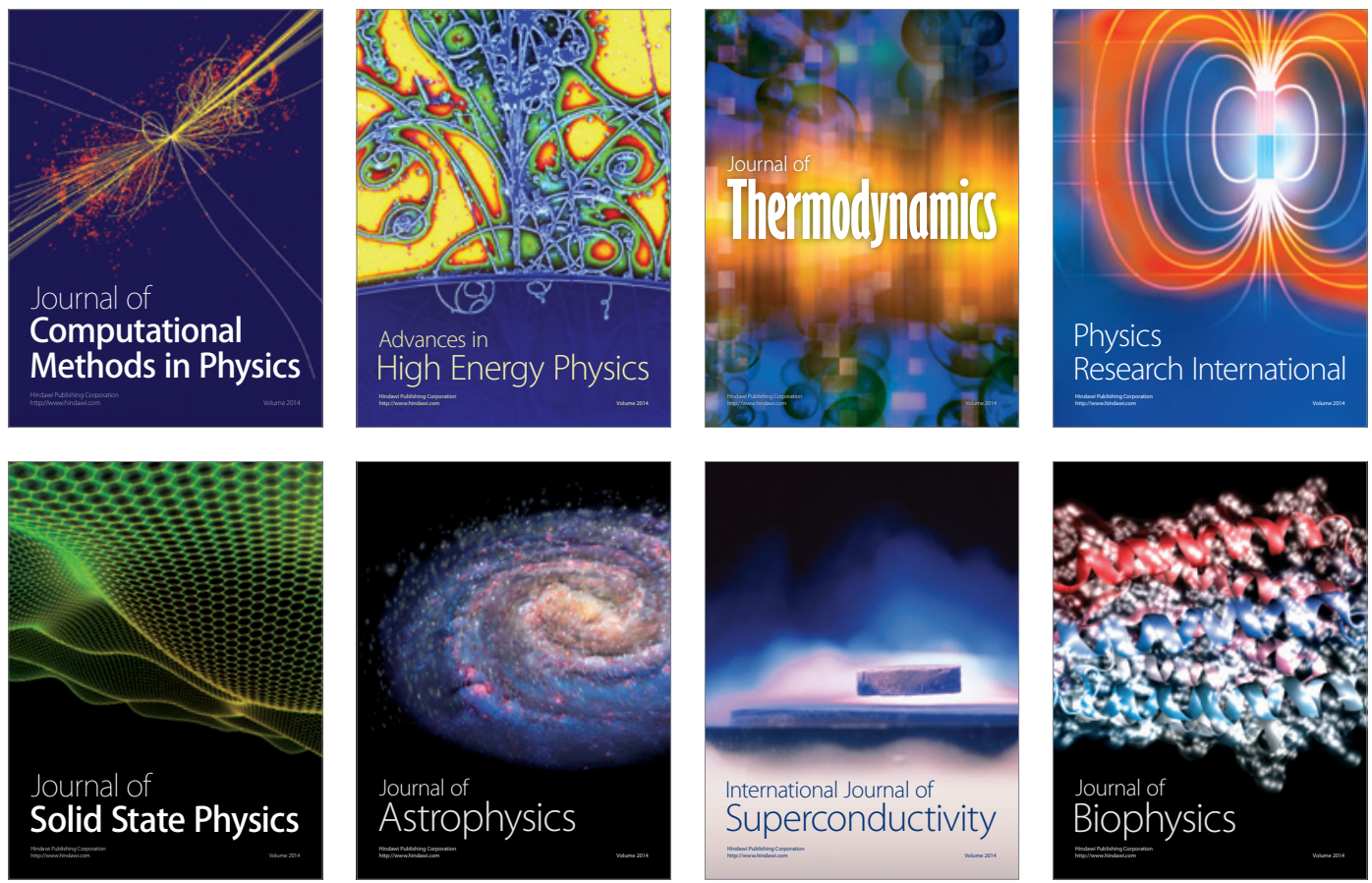
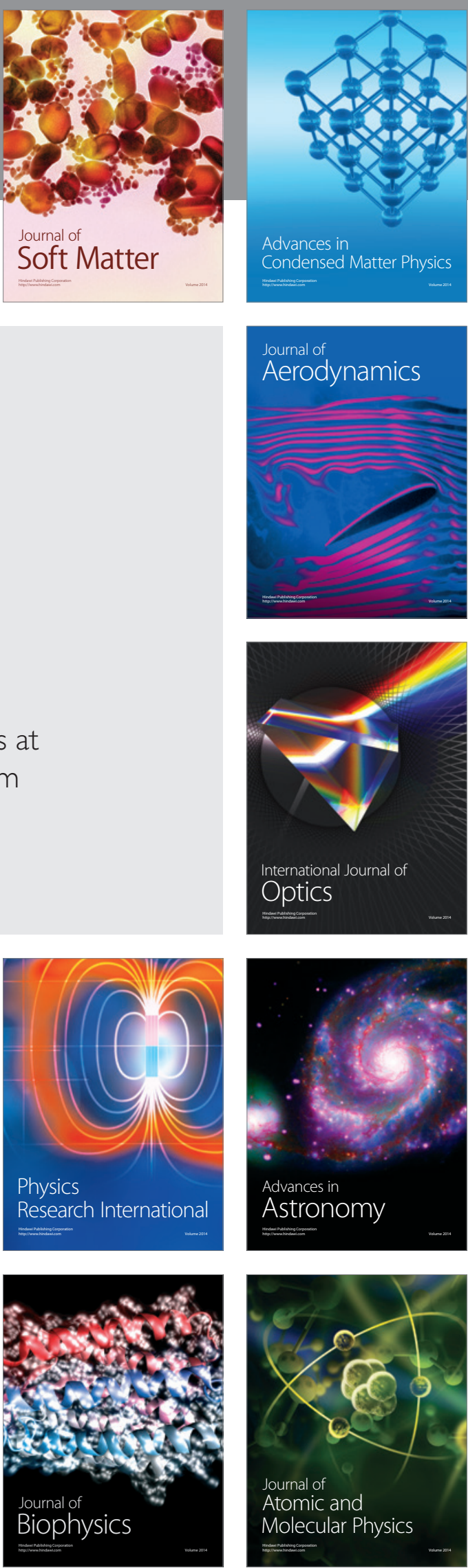